Check for updates

The BMJ

Cite this as: BMJ 2020;371:m3857 http://dx.doi.org/10.1136/bmj.m3857 Published: 02 October 2020

\title{
Covid-19: 120 million rapid tests pledged to low and middle income countries
}

\section{Elisabeth Mahase}

Low and middle income countries have been pledged 120 million covid-19 diagnostic tests, which can provide results in 15 to 30 minutes, by a group of organisations including the Bill and Melinda Gates Foundation and the World Health Organization.

The move comes after WHO approved the first rapid point of care covid-19 test for emergency use-SD Biosensor's test-on 22 September. Several other tests which do not require laboratory processing are also being assessed, including one from Abbott which is currently going through the final stages of approval.

Separate agreements between SD Biosensor, Abbott, and the Bill and Melinda Gates Foundation mean a total of 120 million tests will be made available to low and middle income countries. The price is currently set at $\$ 5$ (€3.9; €4.3) per unit, but WHO said it expects the cost to come down.

The Global Fund, which pools resources to invest in health programmes, has committed an initial \$5om to enable low and middle income countries to get at least 10 million of the tests, with the first orders expected to be placed this week.

The Africa Centres for Disease Control and Prevention, Unitaid, the Clinton Health Access Initiative, and the Foundation for Innovative New Diagnostics are also helping to support countries incorporate these tests into their covid-19 responses. The rollout is expected to begin in up to 20 countries in Africa in October.

The BMJ understands that the UK government is also in discussions with the companies to purchase rapid tests. A spokesperson for the Department of Health and Social Care said, "We are increasing capacity to 500 ooo tests a day by the end of October and the ability to get rapid, on-the-spot results will significantly increase our ability to fight coronavirus."

The test developed by SD Biosensor requires a nasopharyngeal swab and provides results like a pregnancy test, with two lines appearing in the results window of the test device if positive, one control line appearing if the result is negative, and either no lines or only a test line appearing if the test is invalid.

According to the test information sheet, ${ }^{1}$ however, there are limitations. "A negative result may occur if the concentration of antigen or antibody in a specimen is below the detection limit of the test or if the specimen was collected or transported improperly, therefore a negative test result does not eliminate the possibility of SARS-CoV-2 infection, and should be confirmed by viral culture or an molecular assay or ELISA.”

Despite this, WHO has approved the test for emergency use, stating that it is faster and cheaper than laboratory based tests and will enable countries to increase the pace of testing, tracing, and treating people for covid-19, particularly in areas with under-resourced health systems.

Director of the Africa CDC, John Nkengasong, said, “Antigen tests are an important complement to polymerase chain reaction testing, and are crucial to expanding testing capacity throughout Africa. The beauty of antigen testing is that it is fast and gives quick results. It will allow healthcare workers to quickly isolate cases and treat them while tracing their contacts to cut the transmission chain."

The other test that has been promised to low and middle income countries is Abbott's Panbio covid-19 IgG/IgM rapid antigen test. ${ }^{2}$ The test can provide results in 10 to 20 minutes, and uses a fingerstick blood sample. The company claims it has a sensitivity of $96.2 \%$ and specificity of $100.0 \%$. Like the SD Biosensor test, it provides results in a similar way to a pregnancy test and is not able to fully rule out covid-19 infection.

“The test provides preliminary test results. Negative results will not preclude SARS-CoV-2 infection, and they cannot be used as the sole basis for treatment or other management decisions. The test is not intended to be used as a donor screening test for SARS-CoV-2," Abbott's website states.

Biosensor SD. Standard Q covid-19 Ag. http://sdbiosensor.com/xe/product/7672.

2 Panibo covid-19 IgG/IgM rapid test device. unw.gobalpointofcare.abbott/en/product-details/panbio-covid-19-igg-igm-antibody-testhtml. 